\title{
A MCDM approach for evaluating company's environmental practices
}

\author{
R. de Andrés Calle ${ }^{1}$ T. González-Arteaga ${ }^{2}$ L. Martínez ${ }^{3}$ \\ ${ }^{1}$ University of Salamanca \\ ${ }^{2}$ University of Valladolid \\ ${ }^{3}$ University of Jaén
}

\begin{abstract}
The need of organizations to evaluate their environmental practices is increasing, but there are many possibilities to do it. In this paper a decision model is proposed to evaluate company's environmental practice taking into account different collectives of reviewers related to the company's activity. Moreover, this model integrates quantitative and qualitative information with different scales using a multigranular linguistic model that allows to adapt diverse evaluation scales according to the appraisers' knowledge. The final aim is to compute partial and global indicators that can be used by the management team to make their decisions regarding the environmental issues.
\end{abstract}

Keywords: Multi-criteria decision making, environmental performance, non-homogeneous information

\section{Introduction}

With environmental pressures increasing, many companies have begun to give more attention to environmental issues. The ability of organizations to manage their environmental performance is emerging as a strategic issue for firms. They need to implement strategies to reduce their environmental impact. Many studies have been reported about the relation between environmental performance and environmental management systems (see [1]) but the variety of results depend on diverse factors such as goals of a specific company, the operating environment, the management style, etc.

Environmentally conscious practices refer to programs to improve processes and products environmental performance in diverse forms like design for environment, recycling, waste management, life cycle analysis, green supply chain management, environmental certification (ISO 14000), environmental management systems (EMS), etc. They have evolved with influences from reactive and proactive policies taken by organizations (see [2]).

Even though evaluation of environmental performance (EP) is becoming more and more important recently, there is not a total agreement on what environmental performance is or how to measure it.
As a consequence, every organization proposes a different way to define it and to do it (see [1]).

Notwithstanding the evaluation of environmental practices plays a key role in the competitiveness of the companies, many of them either carry out informal ones or do not use it yet. Other companies, keen on this evaluation, use formal methods but based just on the opinion of one or various reviewers, in some cases from top environmental managers, but they hardly ever consider reviewers from company outside. However, it is recognized the importance to include the opinions of other concerned parties (see [3]) to increase internal efficiency and external legitimacy. Then, the results of the evaluation process could not represents correctly the company environmental situation and can be biased and subjective because the evaluation relies on several reviewers that are not the only relevant people to evaluate company's environmental practices (see [4]).

Several multi-criteria decision making approaches have been used as a basis to deal with environmental issues in various studies in the literature. Some examples of such approaches are Analytical Hierarchy Process (AHP) [5], Analytical Network Process (ANP) [6], grey relational analysis [7], Fuzzy TOPSIS (Technique for Order Performance by Similarity to Ideal Solution [8], and others specifically developed models.

Moreover, although there are different types of criteria: quantitative and qualitative, most of the current methods provide only a quantitative precise modelling for their assessments. This fact can produce a lack of precision in the assessments provided by the reviewers for qualitative criteria due to the difficulty of expressing uncertain knowledge in a precise way.

In order to overcome the drawbacks associated with the previous established models to evaluate environmental practices, in this paper we propose a method where different sets of reviewers (internal and external, experts and non-experts) have to evaluate company's environmental practices attending to different criteria and attributes (quantitative and qualitative). Taking into account that the different groups of reviewers may have a different degree of knowledge about the company's environmental practices and the different criteria nature, we then propose a flexible heterogeneous framework in 
which appraisers may express their assessments by means of numerical, interval-valued or linguistic information according to the criteria nature [9]. In addition, we propose the use of the Fuzzy Linguistic Approach [10] to model qualitative indicators, because it provides a direct way to model qualitative information by means of linguistic variables. This approach has been successfully used for this purpose in other evaluation fields and others topics..

Subsequently, the evaluation method should manage this heterogeneous information in order to obtain a global environmental performance indicator. Thus, the problem falls, in a natural way, into the group decision making context. We shall define a multi-criteria model for evaluating company's environmental practices based on classic processes of decision-making.

This paper is organized as follows. In Section 2 we review in short the necessary concepts for our proposal. Section 3 is devoted to introduce the proposed MCDM model for evaluating environmental practices. The paper concludes pointing out some concluding remarks.

\section{Managing non-homogeneous information}

Due to the fact that we consider non-homogenous framework for our evaluation model, in which the appraisers could use numerical, linguistic or interval-valued information. The evaluation model needs to accomplish computations with this type of information and we cannot operate directly with it. We review in short a process presented in [9] to deal with such a type of information that consists in the following steps (see Figure 1):

1. To choose a domain to unify the linguistic information, so-called Basic Linguistic Term Set (BLTS).

2. To conduct the heterogeneous information into the BLTS. First into fuzzy sets and then into linguistic 2-tuples in the BLTS.

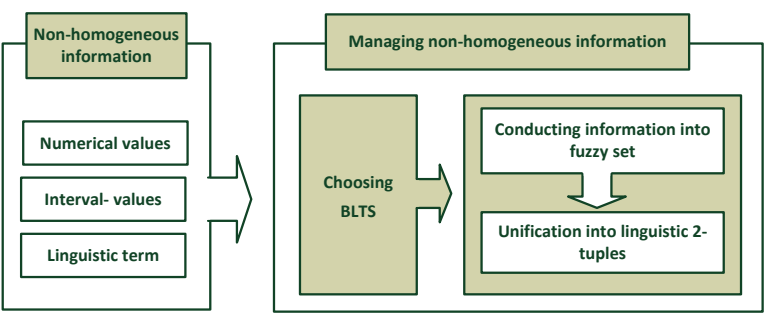

Figure 1: Managing non-homogeneous information

\subsection{Chosing the BLTS}

To deal with non-homogenous information, first it will be conducted in an unique expression domain. This domain will be a linguistic term set called BLTS, noted as $\bar{S}$, that is selected with the aim of keeping as much knowledge as possible (see [9]).

\subsection{Conducting information into fuzzy sets}

Once the BLTS has been chosen in order to accomplish computing processes with non-homogenous information. We will then conduct it in the BLTS by means of fuzzy sets. To do so, we will use the transformation functions presented in [9] from each domain in the heterogeneous framework:

Definition 1 Let $v \in[0,1]$ be a numerical value and $\bar{S}=\left\{\bar{s}_{0}, \bar{s}_{1}, \ldots, \bar{s}_{g}\right\}$ a linguistic term set. The numerical-linguistic transformation function $T_{N \bar{S}}:[0,1] \longrightarrow \mathcal{F}(\bar{S})$ is defined by:

$$
T_{N \bar{S}}(v)=\left\{\left(\bar{s}_{0}, \gamma_{0}\right),\left(\bar{s}_{1}, \gamma_{1}\right), \ldots,\left(\bar{s}_{g}, \gamma_{g}\right)\right\}
$$

with

$$
\gamma_{i}=\mu_{\bar{s}_{i}}(v)=\left\{\begin{array}{l}
0, \text { if } v<a \text { o } v>d \\
\frac{v-a}{b-a}, \text { if } a<v<b \\
1, \text { if } b \leq v \leq c \\
\frac{d-v}{d-c}, \text { if } c<v<d
\end{array}\right.
$$

where $\gamma_{i} \in[0,1]$ and $\mathcal{F}(\bar{S})$ is the set of fuzzy sets on $\bar{S}$, and $\mu_{\bar{s}_{i}}$ is the membership function of the linguistic label $\bar{s}_{i} \in \bar{S}$.

Definition 2 Let $I=[d, e], d \leq e$ be an interval-value in $[0,1]$ and $\bar{S}=\left\{\bar{s}_{0}, \bar{s}_{1}, \ldots, \bar{s}_{g}\right\}$ a linguistic term set. The interval-linguistic transformation function $T_{I \bar{S}}: I \longrightarrow \mathcal{F}(\bar{S})$ is defined by:

$$
T_{I \bar{S}}(I)=\left\{\left(\bar{s}_{0}, \gamma_{0}\right),\left(\bar{s}_{1}, \gamma_{1}\right), \ldots,\left(\bar{s}_{g}, \gamma_{g}\right)\right\}
$$

with

$$
\gamma_{i}=\max _{y} \min \left\{\mu_{I}(y), \mu_{\bar{s}_{i}}(y)\right\}, i=0,1, \ldots, g
$$

where $\mathcal{F}(\bar{S})$ is the set of fuzzy sets on $\bar{S}$, and $\mu_{I}$ and $\mu_{\bar{s}_{i}}$ are the membership functions of the interval-value $I$ and the linguistic label $\bar{s}_{i} \in \bar{S}$, respectively.

Definition 3 Let $S=\left\{s_{0}, s_{1}, \ldots, s_{h}\right\}$ and $\bar{S}=\left\{\bar{s}_{0}, \bar{s}_{1}, \ldots, \bar{s}_{g}\right\}$ be two linguistic term sets, with $h \leq g$. The linguistic transformation function $T_{S \bar{S}}: S \longrightarrow \mathcal{F}(\bar{S})$ is defined by:

$$
T_{S \bar{S}}\left(s_{j}\right)=\left\{\left(\bar{s}_{0}, \gamma_{0}\right),\left(\bar{s}_{1}, \gamma_{1}\right), \ldots,\left(\bar{s}_{g}, \gamma_{g}\right)\right\}
$$

with

$$
\gamma_{i}=\max _{y} \min \left\{\mu_{s_{j}}(y), \mu_{\bar{s}_{i}}(y)\right\}, i=0,1, \ldots, g
$$

where $\mathcal{F}(\bar{S})$ is the set of fuzzy sets on $\bar{S}$, and $\mu_{s_{j}}$ and $\mu_{\bar{s}_{i}}$ are the membership functions of the linguistic labels $s_{j} \in S$ and $\bar{s}_{i} \in \bar{S}$, respectively. 


\subsection{Unification into linguistic 2-tuples}

In order to simplify the computations and improve the understanding of the results, we now transform the fuzzy sets in the BLTS into linguistic 2-tuples in the BLTS [9].

The 2-tuple fuzzy linguistic representation model is based on the concept of symbolic translation [11]. This model represents the linguistic information through a 2-tuple $(s, \alpha)$, where $s$ is a linguistic term and $\alpha$ is a numerical value representation of the symbolic translation [11]. So, being $\beta \in[0, g]$ the value which represents the result of a symbolic aggregation operation, then we can assign a 2-tuple $(s, \alpha)$ that expresses the equivalent information of that given by $\beta$.

Definition 4 Let $S=\left\{s_{0}, \ldots, s_{g}\right\}$ be a set of linguistic terms. The 2-tuple set associated with $S$ is defined as $\langle S\rangle=S \times[-0.5,0.5)$. We define the function $\Delta_{S}:[0, g] \longrightarrow\langle S\rangle$ given by,

$$
\Delta_{S}(\beta)=\left(s_{i}, \alpha\right), \text { with }\left\{\begin{array}{l}
i=\operatorname{round}(\beta), \\
\alpha=\beta-i,
\end{array}\right.
$$

where round assigns to $\beta$ the integer number $i \in$ $\{0,1, \ldots, g\}$ closest to $\beta$.

We note that $\Delta_{S}$ is bijective [11] and $\Delta_{S}^{-1}:\langle S\rangle \longrightarrow[0, g]$ is defined by $\Delta_{S}^{-1}\left(s_{i}, \alpha\right)=i+\alpha$. In this way, the 2 -tuples of $\langle S\rangle$ will be identified with the numerical values in the interval $[0, g]$.

Remark 1 We can consider the injective mapping $S \longrightarrow\langle S\rangle$ that allows us to transform a linguistic term $s_{i}$ into a 2-tuple: $\left(s_{i}, 0\right)$. On the other hand, $\Delta_{S}(i)=\left(s_{i}, 0\right)$ and $\Delta_{S}^{-1}\left(s_{i}, 0\right)=i$, for every $i \in$ $\{0,1, \ldots, g\}$.

The 2-tuple fuzzy linguistic representation model has associated a linguistic computational model [11], that accomplishes processes of CW with symmetrical and triangular-shaped labels in a precise way. Keeping in mind that our objective here is to transform fuzzy sets in the BLTS into linguistic 2tuples, we present the function $\chi$ that carries out this transformation.

Definition 5 Given the linguistic term set $\bar{S}=\left\{\bar{s}_{0}, \bar{s}_{1}, \ldots, \bar{s}_{g}\right\}$, the function $\chi: \mathcal{F}(\bar{S}) \longrightarrow \bar{S} \times[-0.5,0.5)$, is defined by

$\chi\left(\left\{\left(\bar{s}_{0}, \gamma_{0}\right),\left(\bar{s}_{1}, \gamma_{1}\right), \ldots,\left(s_{g}, \gamma_{g}\right)\right\}\right)=\Delta_{\bar{S}}\left(\frac{\sum_{j=0}^{g} j \gamma_{j}}{\sum_{j=0}^{g} \gamma_{j}}\right)$.

\section{An evaluation model for company environmental practices with non-homogeneous information}

In this section, we present our proposal for evaluating company environmental practices based on a classic decision analysis scheme whose accommodation to our problem is showed in Figure 2.

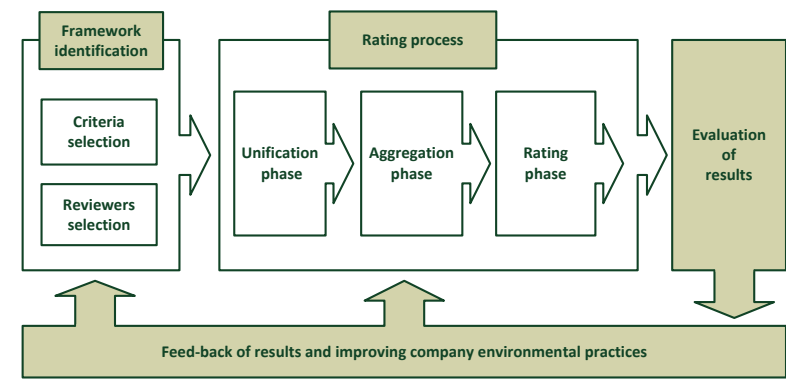

Figure 2: Decision scheme for evaluating company environmental practices

a) Framework identification. In this step of the evaluation process, the criteria and the reviewers of the company's environmental practices are identified.

- Selection of criteria. Environmental performance indicators are used to depict the vast quantity of environmental information of a firm in a comprehensive and concise manner. In the literature, several studies have reported a great variety of indicators or measurement items (see [12] and [13]). Following the standard ISO 14031, in this paper we consider two general categories of EP indicators: management performance indicators (MPI) and operational performance indicators (OPI). The first provides information about management efforts in relation with the policy, people, practices, procedures, decisions and actions at all levels of the organization. The second one provides information about environmental performance of the operations of the organization relative to organization's physical facilities and equipment (material and energy flows). Following these important categories of indicators we can distinguish two types of criteria associated to them: management criteria and operational criteria.

- Selection of reviewers. According to the standard itself [14], in this paper we propose a evaluation method where the company's environmental practices is carried out from diverse collectives related to the activity and not only the technical information recovered from top environmental managers. Moreover, the proposal method distinguish between experts and non-experts reviewers.

b) Rating process. To obtain a global environmental performance indicator of each facility site, all information provided by the appraisers must be aggregated. Due to the fact that the framework is heterogeneous this process is carried out in three phases:

(1) Unification information phase. The heterogenous information provided the different collectives is conducted into a unique expression domain, Basic Linguistic Term Set (BLTS) (see [9]).

(2) Aggregation phase. In this phase the information is aggregated taking into account different 
groups of reviewers and different criteria. We use a multi-step aggregation methodology (see Figure 3 ).

(3) Rating phase. The aim of this phase is ranking evaluated facility sites following the goals established by the Department of Environment.

c) Evaluation of the results. The results of facility sites about environmental practices are taken into account in this stage of the evaluation process due to the fact that one of the most important aspect of an environmental practices evaluation process is providing performance feedback to facility sites.

In the coming subsections we shall present in detail each phase of our model and in order to show how a company or organization could carry out an environmental evaluation process with the proposed model, we provide an example.

\subsection{Evaluation framework}

In order to show how a company could carry out an environmental practices evaluation process, let us suppose a company which wants to evaluate the environmental practices of its facility sites. It is supposed the company has a set of facility sites $X=\left\{x_{1}, \ldots, x_{n}\right\}$ to be evaluated by the following collectives:

- Internal reviewers. This reviewers' collective is made up of:

- A set of company's internal experts, $A^{E}$ :

$$
A^{E}=\left\{a_{1}^{E}, \ldots, a_{m}^{E}\right\} .
$$

- A set of company's internal non-experts such as managers, staff, employees, etc., $A^{N E}$ :

$$
A^{N E}=\left\{a_{1}^{N E}, \ldots, a_{r}^{N E}\right\}
$$

- External reviewers. This reviewers' collective is made up of:

- A set of company's external experts such as auditors, $B^{E}$ :

$$
B^{E}=\left\{b_{1}^{E}, \ldots, b_{s}^{E}\right\} .
$$

- A set of company's external non-experts evaluators which is made up of two different reviewers' collectives:

1. A set of other "stakeholder" (shareholders, suppliers, government regulators, local communities, intermediate customers, large retailers, final consumers), $B^{N E-G}$ :

$$
B^{N E-G}=\left\{b_{1}^{N E-G}, \ldots, b_{t}^{N E-G}\right\} .
$$

2. A set of social constituents (community groups, trade associations, labor unions, environmental groups), $B^{N E-S}$ :

$$
B^{N E-S}=\left\{b_{1}^{N E-S}, \ldots, b_{u}^{N E-S}\right\} .
$$

Moreover, reviewers evaluate company environmental practices attending to two different types of criteria:
- Management performance criteria

$$
Y^{M}=\left\{Y_{1}^{M}, \ldots, Y_{p}^{M}\right\}
$$

- Operational performance criteria

$$
Y^{O}=\left\{Y_{1}^{O}, \ldots, Y_{q}^{O}\right\}
$$

Consequently, there are $p+q$ criteria. As we have mentioned in Section 1, both sets of criteria can have a qualitative or/and quantitative nature.

We notice there are two general sets of criteria to evaluate company environmental practices but each collective of reviewers could only assess the facility site attending to a number of them following the guidelines established by the Department of Environment.

The assessments provided by the members of the different collectives of reviewers $a_{i, j}^{E} \in A^{E}$, $a_{i, j}^{N E} \in A^{N E}, b_{i, j}^{E} \in B^{E}, b_{i, j}^{N E-G} \in B^{N E-G}$ and $b_{i, j}^{N E-S} \in B^{N E-S}$ on the facility site $x_{j}$ according to the criterion $Y_{k}^{-}$are denoted by $a_{i, j, k}^{E}$, $a_{i, j, k}^{N E}, b_{i, j, k}^{E}, b_{i, j, k}^{N E-G}$ and $b_{i, j, k}^{N E-S}$, respectively. Therefore, there are at most $(m+r+s+t+u)(p+q)$ assessments for each locality site by the different reviewers' collectives.

In this paper we consider a evaluation model which mixes quantitative and qualitative criteria. As a consequence, reviewers can express their opinions to each criterion in different domains, depending on the criteria nature and attending to their knowledge about the criterion evaluated. In this way:

- $a_{i, j, k}^{E} \in[0,1]$ or $a_{i, j, k}^{E} \in I=[d, e], I \in[0,1]$ or $a_{i, j, k}^{E} \in S_{A^{E}}^{k}$ for each $i \in\{1, \ldots, m\}$ and each $j \in\{1, \ldots, n\}$.

- $a_{i, j, k}^{N E} \in[0,1]$ or $a_{i, j, k}^{N E} \in I=[d, e], I \in[0,1]$ or $a_{i, j, k}^{N E} \in S_{A^{N E}}^{k}$ for each $i \in\{1, \ldots, r\}$ and each $j \in\{1, \ldots, n\}$.

- $b_{i, j, k}^{E} \in[0,1]$ or $b_{i, j, k}^{E} \in I=[d, e], I \in[0,1]$ or $b_{i, j, k}^{E} \in S_{B^{E}}^{k}$ for each $i \in\{1, \ldots, s\}$ and each $j \in\{1, \ldots, n\}$.

- $b_{i, j, k}^{N E-G} \in[0,1]$ or $b_{i, j, k}^{N E-G} \in I=[d, e], I \in[0,1]$ or $b_{i, j, k}^{N E-G} \in S_{B^{N E-G}}^{k}$ for each $i \in\{1, \ldots, t\}$ and each $j \in\{1, \ldots, n\}$.

- $b_{i, j, k}^{N E-S} \in[0,1]$ or $b_{i, j, k}^{N E-S} \in I=[d, e], I \in[0,1]$ or $b_{i, j, k}^{N E-S} \in S_{B^{N E-S}}^{k}$ for each $i \in\{1, \ldots, u\}$ and each $j \in\{1, \ldots, n\}$.

We can note that any appropriate linguistic term set $S_{-}^{k}$ is characterized by its cardinality or granularity, $\left|S_{-}^{k}\right|$.

Example 1 Let us suppose a company which is carrying out an evaluation on their environmental practices, which involves evaluations from internal and external reviewers. Without loss of generality we consider two facility sites to be evaluated: 
$x_{1}, x_{2}$ according to two qualitative criteria, $Y_{1}, Y_{2}$ and a quantitative criterion $Y_{3}$. For instance, $Y_{1}$ is the extension in pollution control initiatives (management criteria), $Y_{2}$ is "green purchasing" to asses if they incorporate environmental considerations in the purchasing process (management criteria) and $Y_{3}$ is the proportion of recyclable content over the total (operational criteria). The reviewers are:

- Three internal experts $A^{E}=\left\{a_{1}^{E}, a_{2}^{E}, a_{3}^{E}\right\}$.

- Two internal non-experts $A^{N E}=\left\{a_{1}^{N E}, a_{2}^{N E}\right\}$.

- Two external experts $B^{E}=\left\{b_{1}^{E}, b_{2}^{E}\right\}$.

- Three external non-experts from stakeholder $B^{N E-G}=\left\{b_{1}^{N E-G}, b_{2}^{N E-G}, b_{3}^{N E-G}\right\}$.

- Two external non-experts from social constituents $B^{N E-S}=\left\{b_{1}^{N E-S}, b_{2}^{N E-S}\right\}$.

Each group of reviewers uses their own balanced linguistic term sets $S_{-}^{k}$ to provide their knowledge of every qualitative criteria and the interval $[0,1]$ for the quantitative one. The term sets referred to the first criterion $\left(S_{A^{E}}^{1} S_{A^{N E}}^{1}, S_{B^{E}}^{1} S_{B^{N E-G}}^{1} S_{B^{N E-S}}^{1}\right)$ have 5 linguistic terms and the term sets referred to the second criterion $\left(S_{A^{E}}^{2} S_{A^{N E}}^{2}, S_{B^{E}}^{2} S_{B^{N E-G}}^{2}\right.$ $\left.S_{B^{N E-S}}^{2}\right)$ have 7 linguistic terms. The associated semantics are included in the following Table 1.

Table 1: Label sets for the criteria

Label set for criterion $Y_{1}$

\begin{tabular}{|c|c|c|}
\hline$s_{0}^{1}$ & Very poor & $(0,0,0.25)$ \\
\hline$s_{1}^{1}$ & Poor & $(0,0.25,0.5)$ \\
\hline$s_{2}^{1}$ & Fair & $(0.25,0.5,0.75)$ \\
\hline$s_{3}^{1}$ & Good & $(0.5,0.75,1)$ \\
\hline$s_{4}^{1}$ & Very good & $(0.75,1,1)$ \\
\hline \multicolumn{3}{|c|}{ Label set for criterion $Y_{2}$} \\
\hline$s_{0}^{2}$ & Never & $(0,0,0.16)$ \\
\hline$s_{1}^{2}$ & Very Rarely & $(0,0.16,0.34)$ \\
\hline$s_{2}^{2}$ & Rarely & $(0.16,0.34,0.5)$ \\
\hline$s_{3}^{2}$ & Sometimes & $(0.34,0.5,0.66)$ \\
\hline$s_{4}^{2}$ & Frequently & $(0.5,0.66,0.84)$ \\
\hline$s_{5}^{2}$ & Very Frequently & $(0.66,0.84,1)$ \\
\hline$s_{6}^{2}$ & Always & $(0.84,1,1)$ \\
\hline
\end{tabular}

First of all and once the evaluation framework has been fixed the reviewers express their opinions about each facility site. In Table 2 is indicated the assessments provided by the appraisers about facility sites $x_{1}$ and $x_{2}$, for each criterion.

\subsection{Rating process}

To operate with heterogeneous information, it is followed the process reviewed in Section 2. Its adaption to the current problem is detailed below.

\subsubsection{Unification phase}

Dealing with with heterogeneous information provided by the different collectives assessed in differ-
Table 2: Reviewers' assessments for each facility site and each criterion

\begin{tabular}{|c|c|c|c|c|c|c|}
\hline & \multicolumn{3}{|c|}{$x_{1}$} & \multicolumn{3}{|c|}{$x_{2}$} \\
\hline & $Y_{1}$ & $Y_{2}$ & $Y_{3}$ & $Y_{1}$ & $Y_{2}$ & $Y_{3}$ \\
\hline$a_{1}^{E}$ & $s_{1}^{1}$ & $s_{5}^{2}$ & 0.30 & $s_{1}^{1}$ & $s_{4}^{2}$ & 0.60 \\
\hline$a_{2}^{E}$ & $s_{1}^{1}$ & $s_{5}^{2}$ & 0.27 & $s_{1}^{1}$ & $s_{5}^{2}$ & 0.65 \\
\hline$a_{3}^{E}$ & $s_{2}^{1}$ & $s_{4}^{2}$ & 0.30 & $s_{0}^{1}$ & $s_{5}^{2}$ & 0.65 \\
\hline & $s_{1}^{1}$ & $s_{4}^{2}$ & - & $s_{0}^{1}$ & $s_{5}^{2}$ & - \\
\hline$a_{2}^{\bar{N} E}$ & $s_{2}^{1}$ & $s_{4}^{2}$ & - & $s_{1}^{1}$ & $s_{4}^{2}$ & - \\
\hline$b_{1}^{E}$ & $s_{3}^{1}$ & $s_{5}^{2}$ & 0.25 & $s_{1}^{1}$ & $s_{4}^{2}$ & 0.52 \\
\hline$b_{2}^{E}$ & $s_{1}^{1}$ & $s_{4}^{2}$ & 0.20 & $s_{0}^{1}$ & $s_{3}^{2}$ & 0.55 \\
\hline$b_{1}^{N E-G}$ & $s_{1}^{1}$ & $s_{5}^{2}$ & - & $s_{1}^{1}$ & $s_{5}^{2}$ & - \\
\hline$b_{2}^{N E-G}$ & $s_{2}^{1}$ & $s_{3}^{2}$ & - & $s_{1}^{1}$ & $s_{4}^{2}$ & - \\
\hline$b_{3}^{N E-G}$ & $s_{2}^{1}$ & $s_{4}^{2}$ & - & $s_{0}^{1}$ & $s_{5}^{2}$ & - \\
\hline$b_{1}^{N E-S}$ & $s_{2}^{1}$ & $s_{5}^{2}$ & - & $s_{2}^{1}$ & $s_{5}^{2}$ & - \\
\hline$b_{2}^{N E-S}$ & $s_{2}^{1}$ & $s_{5}^{2}$ & - & $s_{1}^{1}$ & $s_{5}^{2}$ & - \\
\hline
\end{tabular}

ent domains (numerical, interval-valued and linguistic), first it is conducted the non-homogeneous information into an unique expression domain, BLTS $[9], \bar{S}=\left\{\bar{s}_{0}, \bar{s}_{1}, \ldots, \bar{s}_{g}\right\}$ (see Figure 1 ). Once the BLTS has been chosen, the non-homogeneous information for each criterion $Y_{k}^{-}$is unified by means of fuzzy sets in $\bar{S}$ using the functions $T_{N \bar{S}}, T_{I \bar{S}}$ and $T_{S \bar{S}}$ (Definitions 1,2 and 3).The information is then expressed into an unique linguistic term set, through fuzzy sets in $\bar{S}$.

In order to facilitate the aggregation process and the understandability of the results, we transform the fuzzy sets in $\bar{S}$ into linguistic 2-tuples using the functions $\chi$ and $\Delta$ presented in Definitions 4 and 5 , respectively. The transformed assessments for each collective are then $\bar{a}_{i, j, k}^{E} \in\langle\bar{S}\rangle$ for internal experts reviewers, $\bar{a}_{i, j, k}^{N E} \in\langle\bar{S}\rangle$ for internal non-experts reviewers, $\bar{b}_{i, j, k}^{E} \in\langle\bar{S}\rangle$ for external experts reviewers and $\bar{b}_{i, j, k}^{N E-G} \in\langle\bar{S}\rangle$ and $\bar{b}_{i, j, k}^{N E-S} \in\langle\bar{S}\rangle$ for external non-experts reviewers.

We can note that all the information provided by the different collectives has already unified into 2tuples in the BLTS. Therefore, we can operate in a symbolic way to obtain the appraisal results.

Example 2 Following Example 1, the assessments provided by the reviewers will be conducted into a unique linguistic term set, BLTS. In this case, we will consider that the BLTS is $\bar{S}=\left\{\bar{s}_{0}, \ldots, \bar{s}_{6}\right\}$. To transform the input information into $F(\bar{S})$, we apply the transformation function from Definitions 2, 1 and 3. When all information is expressed by means of fuzzy sets defined in the BLTS, we transform every fuzzy set in $\mathrm{S}$ into a linguistic 2-tuple (see Definition 5). The results of these transformations are shown in Table 3.

\subsubsection{Aggregation phase}

To obtain a global environmental performance indicator of each facility site, we are carried out an ag- 
Table 3: Reviewers' transformed-assessments for each facility site and each criterion

\begin{tabular}{cccc} 
& \multicolumn{3}{c}{$x_{1}$} \\
\cline { 2 - 4 } & $Y_{1}$ & $Y_{2}$ & $Y_{3}$ \\
\hline$a_{1}^{E}$ & $\left(\bar{s}_{2},-0.5\right)$ & $\left(\bar{s}_{5}, 0\right)$ & $\left(\bar{s}_{2},-0.22\right)$ \\
$a_{2}^{E}$ & $\left(\bar{s}_{2},-0.5\right)$ & $\left(\bar{s}_{5}, 0\right)$ & $\left(\bar{s}_{2},-0.39\right)$ \\
$a_{3}^{E}$ & $\left(\bar{s}_{3},-0.17\right)$ & $\left(\bar{s}_{4}, 0\right)$ & $\left(\bar{s}_{2},-0.22\right)$ \\
$a_{1}^{N E}$ & $\left(\bar{s}_{2},-0.5\right)$ & $\left(\bar{s}_{4}, 0\right)$ & - \\
$a_{2}^{N E}$ & $\left(\bar{s}_{3},-0.17\right)$ & $\left(\bar{s}_{4}, 0\right)$ & - \\
$b_{1}^{E}$ & $\left(\bar{s}_{5},-0.49\right)$ & $\left(\bar{s}_{5}, 0\right)$ & $\left(\bar{s}_{3}, 0.31\right)$ \\
$b_{2}^{E}$ & $\left(\bar{s}_{2},-0.5\right)$ & $\left(\bar{s}_{4}, 0\right)$ & $\left(\bar{s}_{1}, 0.22\right)$ \\
$b_{1}^{N E-G}$ & $\left(\bar{s}_{2},-0.5\right)$ & $\left(\bar{s}_{5}, 0\right)$ & - \\
$b_{2}^{N E-G}$ & $\left(\bar{s}_{3},-0.17\right)$ & $\left(\bar{s}_{3}, 0\right)$ & - \\
$b_{3}^{N E-G}$ & $\left(\bar{s}_{3},-0.17\right)$ & $\left(\bar{s}_{4}, 0\right)$ & - \\
$b_{1}^{N E-S}$ & $\left(\bar{s}_{3},-0.17\right)$ & $\left(\bar{s}_{5}, 0\right)$ & - \\
$b_{2}^{N E-S}$ & $\left(\bar{s}_{3},-0.17\right)$ & $\left(\bar{s}_{5}, 0\right)$ & - \\
\hline
\end{tabular}

\begin{tabular}{cccc} 
& \multicolumn{3}{c}{$x_{2}$} \\
\cline { 2 - 4 } & $Y_{1}$ & $Y_{2}$ & $Y_{3}$ \\
\hline$a_{1}^{E}$ & $\left(\bar{s}_{2},-0.5\right)$ & $\left(\bar{s}_{4}, 0\right)$ & $\left(\bar{s}_{4},-0.38\right)$ \\
$a_{2}^{E}$ & $\left(\bar{s}_{2},-0.5\right)$ & $\left(\bar{s}_{5}, 0\right)$ & $\left(\bar{s}_{4},-0.06\right)$ \\
$a_{3}^{E}$ & $\left(\bar{s}_{1},-0.40\right)$ & $\left(\bar{s}_{5}, 0\right)$ & $\left(\bar{s}_{4},-0.06\right)$ \\
$a_{1}^{N E}$ & $\left(\bar{s}_{1},-0.40\right)$ & $\left(\bar{s}_{5}, 0\right)$ & - \\
$a_{2}^{N E}$ & $\left(\bar{s}_{2},-0.5\right)$ & $\left(\bar{s}_{4}, 0\right)$ & - \\
$b_{1}^{E}$ & $\left(\bar{s}_{2},-0.5\right)$ & $\left(\bar{s}_{4}, 0\right)$ & $\left(\bar{s}_{3}, 0.13\right)$ \\
$b_{2}^{E}$ & $\left(\bar{s}_{1},-0.40\right)$ & $\left(\bar{s}_{3}, 0\right)$ & $\left(\bar{s}_{3}, 0.32\right)$ \\
$b_{1}^{N E-G}$ & $\left(\bar{s}_{2},-0.5\right)$ & $\left(\bar{s}_{5}, 0\right)$ & - \\
$b_{2}^{N E-G}$ & $\left(\bar{s}_{2},-0.5\right)$ & $\left(\bar{s}_{4}, 0\right)$ & - \\
$b_{3}^{N E-G}$ & $\left(\bar{s}_{1},-0.40\right)$ & $\left(\bar{s}_{5}, 0\right)$ & - \\
$b_{1}^{N E-S}$ & $\left(\bar{s}_{3},-0.17\right)$ & $\left(\bar{s}_{5}, 0\right)$ & - \\
$b_{2}^{N E-S}$ & $\left(\bar{s}_{2},-0.5\right)$ & $\left(\bar{s}_{5}, 0\right)$ & - \\
\hline
\end{tabular}

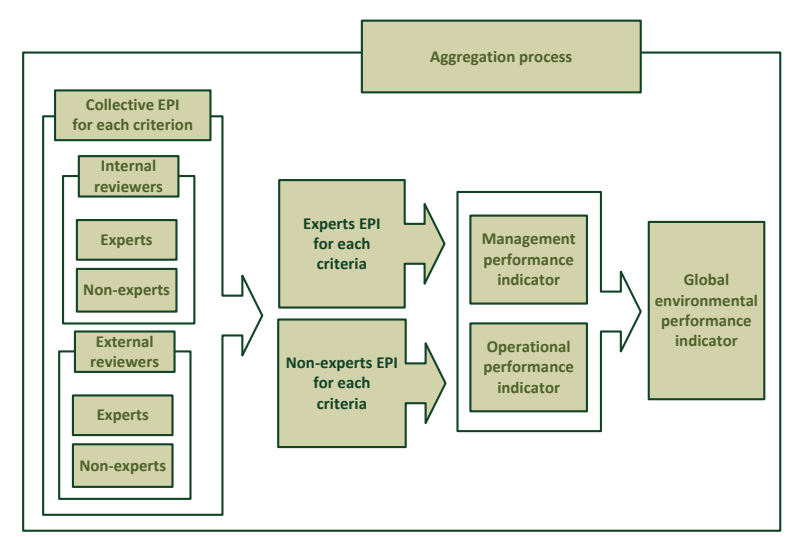

Figure 3: Steps of the aggregation phase

gregation method which has several steps (see Figure 3). To do so, individual assessments will be aggregated by using aggregation operators that allow to operate in a symbolic way and have good properties. So, the use of 2-tuples OWA operator seems suitable (see [11]).
Definition 6 Let $\left(\left(l_{1}, \alpha_{1}\right), \ldots,\left(l_{m}, \alpha_{m}\right)\right) \in\langle\bar{S}\rangle^{m}$ be a vector of linguistic 2-tuples, and $\boldsymbol{w}=$ $\left(w_{1}, \ldots, w_{m}\right) \in[0,1]^{m}$ be a weighting vector such that $\sum_{i=1}^{m} w_{i}=1$. The 2-tuple $O W A$ operator associated with $\boldsymbol{w}$ is the function $G^{w}:\langle\bar{S}\rangle^{m} \longrightarrow\langle\bar{S}\rangle$ defined by

$$
G^{w}\left(\left(l_{1}, \alpha_{1}\right), \ldots,\left(l_{m}, \alpha_{m}\right)\right)=\Delta_{\bar{S}}\left(\sum_{i=1}^{m} w_{i} \beta_{i}^{*}\right)
$$

where $\beta_{i}^{*}$ is the $i$-th largest element of $\left\{\Delta_{\bar{S}}^{-1}\left(l_{1}, \alpha_{1}\right), \ldots, \Delta_{\bar{S}}^{-1}\left(l_{m}, \alpha_{m}\right)\right\}$.

Remark 2 2-tuple OWA operator satisfies some interesting properties as compensativeness, idempotency, symmetry and monotonicity.

There are different methods to compute the weighting vectors. Yager suggested an interesting way to compute the weighting vector for OWA operators using non-decreasing linguistic quantifiers (see [15]), that we shall use in our model. Some examples of non-decreasing relative linguistic quantifiers are "most", "at least half" and "as many as possible".

Following, we present in further detail each stage in the aggregation process. It is important to note that each aggregation procedure can use a different linguistic quantifier.

1. Computing environmental performance indicators for each reviewers' collective and each criterion.

The reviewers' assessments are aggregated for each criterion and each collective by means of a 2-tuple OWA operator, $G_{-}^{-}$. Then, for each collective and for every criterion $Y_{k}^{-}$, the process is conducted in the following manner.

- For internal reviewers (experts and nonexperts, respectively):

$$
\begin{aligned}
I_{k}^{A^{E}}\left(x_{j}\right) & =G_{k}^{A^{E}}\left(\bar{a}_{1, j, k}^{E}, \ldots, \bar{a}_{m, j, k}^{E}\right), \\
I_{k}^{A^{N E}}\left(x_{j}\right) & =G_{k}^{A^{N E}}\left(\bar{a}_{1, j, k}^{N E}, \ldots, \bar{a}_{r, j, k}^{N E}\right) .
\end{aligned}
$$

- For external reviewers (experts and nonexperts, respectively):

$$
\begin{aligned}
I_{k}^{B^{E}}\left(x_{j}\right) & =G_{k}^{B^{E}}\left(\bar{b}_{1, j, k}^{E}, \ldots, \bar{b}_{s, j, k}^{E}\right), \\
I_{k}^{B^{N E}}\left(x_{j}\right) & =G_{k}^{B^{N E}}\left(I_{k}^{B^{N E-G}}\left(x_{j}\right), I_{k}^{B^{N E-S}}\left(x_{j}\right)\right),
\end{aligned}
$$

where $I_{k}^{B^{N E-G}}\left(x_{j}\right)$ is the environmental performance indicator for stakeholder reviewers:

$I_{k}^{B^{N E-G}}\left(x_{j}\right)=G_{k}^{B^{N E-G}}\left(\bar{b}_{1, j, k}^{N E-G}, \ldots, \bar{b}_{t, j, k}^{N E-G}\right)$

and $I_{k}^{B^{N E-S}}\left(x_{j}\right)$ is the environmental performance indicator for social reviewers: $I_{k}^{B^{N E-S}}\left(x_{j}\right)=G_{k}^{B^{N E-S}}\left(\bar{b}_{1, j, k}^{N E-S}, \ldots, \bar{b}_{u, j, k}^{N E-S}\right)$. 
2. Computing environmental performance indicators for experts/non-experts reviewers and each criterion.

The previous environmental performance indicators for the $x_{j}$ facility site: $I_{k}^{A^{E}}\left(x_{j}\right)$, $I_{k}^{A^{N E}}\left(x_{j}\right), I_{k}^{B^{E}}\left(x_{j}\right)$ and $I_{k}^{B^{N E}}\left(x_{j}\right)$ are aggregated for each criterion taking into account if the reviewers are experts or not. Then, the previous indicators belonging to the experts reviewers are aggregated by means of a 2-tuple OWA operator:

$$
G_{k}^{E}:\langle\bar{S}\rangle^{2} \longrightarrow\langle\bar{S}\rangle
$$

obtaining a 2-tuple over the BLTS for each criterion $Y_{k}^{-}$:

$$
I_{k}^{E}\left(x_{j}\right)=G_{k}^{E}\left(I_{k}^{A^{E}}\left(x_{j}\right), I_{k}^{B^{E}}\left(x_{j}\right)\right) \in\langle\bar{S}\rangle .
$$

Analogously to the experts reviewers, an environmental performance indicator is computed for each criterion $Y_{k}^{-}$by aggregating the opinions of all non-experts reviewers:

$$
G_{k}^{N E}:\langle\bar{S}\rangle^{2} \longrightarrow\langle\bar{S}\rangle
$$

obtaining a 2-tuple over the BLTS for each criterion $Y_{k}^{-}$:

$$
I_{k}^{N E}\left(x_{j}\right)=G_{k}^{N E}\left(I_{k}^{A^{N E}}\left(x_{j}\right), I_{k}^{B^{N E}}\left(x_{j}\right)\right) \in\langle\bar{S}\rangle .
$$

3. Computing management and operational performance indicators.

They are obtained by means of aggregating the previous experts and non-experts indicators taking into account the two types of criteria: management and operational.

- Management performance indicator. In order to calculate this indicator we aggregate the experts and non-experts indicators for management criteria, $Y^{M}=\left\{Y_{1}^{M}, \ldots, Y_{p}^{M}\right\}$ by means of a 2 tuple OWA operator:

$$
G^{M}:\langle\bar{S}\rangle^{p} \longrightarrow\langle\bar{S}\rangle
$$

obtaining a 2-tuple over the BLTS:

$$
\begin{array}{r}
I^{M}\left(x_{j}\right)=G^{M}\left(I_{1}^{E}\left(x_{j}\right), \ldots, I_{p}^{E}\left(x_{j}\right),\right. \\
\left.I_{1}^{N E}\left(x_{j}\right) \ldots, I_{p}^{N E}\left(x_{j}\right)\right) \in\langle\bar{S}\rangle .
\end{array}
$$

- Operational performance indicator. Analogously to management performance indicator, an operational performance indicator is computed for operational criteria, $Y^{O}=\left\{Y_{1}^{O}, \ldots, Y_{q}^{O}\right\}$ by aggregating the experts and no-experts indicators for such criteria:

$$
G^{O}:\langle\bar{S}\rangle^{q} \longrightarrow\langle\bar{S}\rangle
$$

obtaining a 2-tuple over the BLTS:

$$
\begin{array}{r}
I^{O}\left(x_{j}\right)=G^{O}\left(I _ { 1 } ^ { E } \left(x_{j}, \ldots, I_{q}^{E}\left(x_{j}\right),\right.\right. \\
\left.I_{1}^{N E}\left(x_{j}\right), \ldots, I_{q}^{N E}\left(x_{j}\right)\right) \in\langle\bar{S}\rangle .
\end{array}
$$

4. Computing a global environmental performance indicator.

It is obtained by aggregating the management and the operational performance indicators, by means of a 2-tuple OWA operator

$$
G:\langle\bar{S}\rangle^{2} \longrightarrow\langle\bar{S}\rangle
$$

obtaining a 2-tuple in the BLTS:

$$
I\left(x_{j}\right)=G\left(I^{M}\left(x_{j}\right), I^{O}\left(x_{j}\right)\right) \in\langle\bar{S}\rangle .
$$

All the indicators obtained in each step of the aggregation process, $I_{k}^{A^{E}}\left(x_{j}\right), I_{k}^{A^{N E}}\left(x_{j}\right), I_{k}^{B^{E}}\left(x_{j}\right)$, $I_{k}^{B^{N E-G}}\left(x_{j}\right), I_{k}^{B^{N E-S}}\left(x_{j}\right), I_{k}^{E}\left(x_{j}\right), I_{k}^{N E}\left(x_{j}\right), I^{M}\left(x_{j}\right)$, $I^{O}\left(x_{j}\right)$ and $I$ are used for evaluating company environmental practices.

\subsubsection{Rating phase}

In the exploitation phase, the management team of the Department of Environment shall classify and order facility sites according to the environmental performance indicators obtained in the previous phase. For sorting facility sites within the set of linguistic categories of the BLTS, we only need to take into account the first component of the 2-tuples obtained in each stage of the aggregation phase. However, for ranking facility sites it should be necessary to take into account the two components of the corresponding 2-tuples. The process of pairwise comparison among these values expressed by linguistic 2 -tuples is carried out according to the ordinary lexicographic order presented in [11].

\subsection{Evaluation of results and feed-back}

Few models of evaluation environmental practices take into account in the decision making process the evaluation of the results from the rating phase. Our proposal includes the evaluation of the results into the decision making process with the purpose of improving the environmental practices, knowing facility sites' weak points and synchronizing environmental facility sites' goals with company's environmental goals (see Figure 2). This part of evaluation environmental procedure is so-called feedback process.

Example 3 Following Examples 1 and 2, the weighting vectors used in each stage of the aggregation procedure are determined by a fuzzy linguistic quantifier. Particularly, in this example we use the quantifier "most" whose parameters are $(0.3,0.8)$ to aggregate information in all stages of the aggregation procedure. The aggregation process computes 
Table 4: Global performance values for each facility site

$$
\begin{gathered}
I\left(x_{1}\right)=\left(\bar{s}_{2}, 0.01\right) \\
I\left(x_{2}\right)=\left(\bar{s}_{3},-0.23\right)
\end{gathered}
$$

different values to obtain the global environmental performance indicator for each facility site (see Table 4).

Taking into account the result of the last stage of the ranking phase and the ordinary lexicographic order on $\langle\bar{S}\rangle$, the facility site $x_{2}$ is the best carrying out environmental practices. The Department of Environment now knows the weak points of the facility site $x_{1}$ and it will try to synchronize environmental facility site' goals with company's environmental goals.

\section{Conclusions}

The evaluation of environmental practices is a process that allows companies and organizations to determine their efficiency and effectiveness of their environmental practices. In this contribution we have presented a MCDM model, taking into account that appraisers are expressing objective and subjective perceptions and might present different degrees of knowledge about evaluated facility sites. Thus, in our proposal appraisers could express their assessments in different scales according to their knowledge and criteria nature. The presented model not only obtain a global environmental performance indicator for each facility site, but also it obtains intermediate environmental performance indicators according to the opinions of each set of reviewers and criterion, and a management and operational performance indicators. All these results are expressed in a linguistic way in order to improve the understanding of such results to all the people involved in the evaluation process. It is worth emphasizing that the proposed model is quite flexible and it allows to the management team customizes how to aggregate the individual opinions.

\section{Acknowledgements}

This paper has been partially supported by the research projects: TIN2009-08286, Spanish Ministerio de Educación y Ciencia (Project ECO2009-07332), and ERDF.

\section{References}

[1] D. Nawrocka and T. Parker. Finding the connection: environmental management systems and environmental performance. Journal of Cleaner Production, 17:601-607, 2009.
[2] B. Lin, C.A. Jones, and C. Hsieh. Environmental practices and assessment: a process perspective. Industrial Management and Data Systems, 101(2):71-79, 2001.

[3] N. Darnall, I. Henriques, and P. Sadorsky. Do environmental management systems improve business performance in an international setting. Journal of International Management, 14:364-376, 2008.

[4] N. Darnall, I. Seol, and J. Sarkis. Perceived stakeholder influences and organizations' use of environmental audits. Accounting, Organizations and Society, 34:170-187, 2009.

[5] A.H.I. Lee, H. Kang, C. Hsu, and H. Hung. A green supplier selection model for high-tech industry. Expert Systems with Applications, 36:7917-7927, 2009.

[6] J. Sarkis. A strategic decision framework for green supply chain management. Journal of Cleaner Production, 11:397-409, 2003.

[7] M.L. Tseng and A.S.F. Chiu. Evaluating firm's green supply chain management in linguistic preferences. Journal of Cleaner Production, In press:1-10, 2010.

[8] A. Awasthi, S.S. Chauhan, and S.K. Goyal. A fuzzy multicriteria approach for evaluatin environmental performance of suppliers. International Journal of Production Economics, 126:370-378, 2010.

[9] F. Herrera, L. Martínez, and P. J. Sánchez. Managing non-homogeneous information in group decision making. European Journal of Operational Research, 166:115-132, 2005.

[10] L. Zadeh. The concept of a linguistic variable and its applications to approximate reasoning. Information Sciences, Part I and Part II (8), Part III (9):199-249,301-357,43-80, 1975.

[11] F. Herrera and L. Martínez. A 2-tuple fuzzy linguistic representation model for computing with words. IEEE Transactions on Fuzzy Systems, 8:746-752, 2000.

[12] P.K. Humphreys, Y.K. Wong, and F.T.S. Chan. Integrating environmental criteria into the supplier selection process. Journal of Materials Processing Technology, 138:349-356, 2003.

[13] Q. Zhu, J. Sarkis, and K. Laic. Confirmation of a measurement model for green supply chain management practices implementation. Int. J. Production Economics, 111:261-273, 2008.

[14] ISO. Environmental management systems - requirements with guidance for use (ISO 14001: 2004). 2004.

[15] R. R. Yager. On ordered weighted averaging operators in multicriteria decision making. IEEE Transactions on Systems, Man, and Cybernetics, 18:183-190, 1988. 